\title{
Malignancy Rate in Thyroid Nodules Classified as Bethesda Category III (AUS/FLUS)
}

\author{
Allen S. Ho, ${ }^{1}$ Evan E. Sarti, ${ }^{1}$ Kunal S. Jain, ${ }^{1}$ Hangjun Wang, ${ }^{2}$ Iain J. Nixon, ${ }^{1}$ Ashok R. Shaha, \\ Jatin P. Shah, ${ }^{1}$ Dennis H. Kraus, ${ }^{3}$ Ronald Ghossein, ${ }^{2}$ Stephanie A. Fish, ${ }^{4}$ Richard J. Wong, ${ }^{1}$ \\ Oscar Lin, ${ }^{2}$ and Luc G.T. Morris ${ }^{1}$
}

Background: The Bethesda System for Reporting Thyroid Cytopathology is the standard for interpreting fine needle aspiration (FNA) specimens. The "atypia of undetermined significance/follicular lesion of undetermined significance" (AUS/FLUS) category, known as Bethesda Category III, has been ascribed a malignancy risk of $5-15 \%$, but the probability of malignancy in AUS/FLUS specimens remains unclear. Our objective was to determine the risk of malignancy in thyroid FNAs categorized as AUS/FLUS at a comprehensive cancer center. Methods: The management of 541 AUS/FLUS thyroid nodule patients treated at Memorial Sloan-Kettering Cancer Center between 2008 and 2011 was analyzed. Clinical and radiologic features were examined as predictors for surgery. Target AUS/FLUS nodules were correlated with surgical pathology.

Results: Of patients with an FNA initially categorized as AUS/FLUS, 64.7\% (350/541) underwent immediate surgery, $17.7 \%$ (96/541) had repeat FNA, and 17.6\% (95/541) were observed. Repeat FNA cytology was unsatisfactory in $5.2 \%(5 / 96)$, benign in $42.7 \%$ (41/96), AUS/FLUS in $38.5 \%$ (37/96), suspicious for follicular neoplasm in $5.2 \%$ (5/96), suspicious for malignancy in $4.2 \%$ (4/96), and malignant in $4.2 \%$ (4/96). Of nodules with two consecutive AUS/FLUS diagnoses that were resected, 26.3\% (5/19) were malignant. Among all index AUS/FLUS nodules (triaged to surgery, repeat FNA, or observation), malignancy was confirmed on surgical pathology in 26.6\% [CI 22.4-31.3]. Among AUS/FLUS nodules triaged to surgery, the malignancy rate was $37.8 \%$ [CI 33.1-42.8]. Incidental cancers were found in $22.3 \%$ of patients. On univariate logistic regression analysis, factors associated with triage to surgery were younger patient age $(p<0.0001)$, increasing nodule size $(p<0.0001)$, and nodule hypervascularity $(p=0.032)$.

Conclusions: In patients presenting to a comprehensive cancer center, malignancy rates in nodules with AUS/ FLUS cytology are higher than previously estimated, with 26.6-37.8\% of AUS/FLUS nodules harboring cancer. These data imply that Bethesda Category III nodules in some practice settings may have a higher risk of malignancy than traditionally believed, and that guidelines recommending repeat FNA or observation merit reconsideration.

\section{Introduction}

$\mathbf{F}$ INE-NEEDLE ASPIRATION (FNA) plays an instrumental role in the workup of the thyroid nodule by estimating the risk of malignancy and thereby assisting rational triage of patients to surgery or observation. While only approximately $5 \%$ of thyroid nodules harbor malignancy, the use of FNA has permitted highly effective risk stratification, such that more than $50 \%$ of surgically resected nodules ultimately reveal cancer (1). Accordingly, this has effectively reduced the number of unnecessary surgeries and their associated sequelae.
Despite the benefits of FNA, cytopathology reports in the past have been ambiguous or difficult to interpret. Vague descriptors such as "atypical," "indeterminate," or "cannot exclude" have been demonstrated to confuse management (2). These terms are perceived differently by pathologists, endocrinologists, and surgeons, especially between institutions $(3,4)$. Such shortcomings were the impetus for the Bethesda System for Reporting Thyroid Cytopathology, which standardized thyroid FNA results into six diagnostic categories and has become the accepted mode of communication between the various specialties caring for thyroid nodule patients $(5,6)$. Implementation of the Bethesda

\footnotetext{
${ }^{1}$ Head and Neck Service, Department of Surgery; ${ }^{2}$ Department of Pathology; ${ }^{4}$ Endocrinology Service, Department of Medicine; Memorial Sloan-Kettering Cancer Center, New York, New York.

${ }^{3}$ New York Head and Neck Institute, NorthShore-LIJ Health System, New York, New York.
} 
System for Reporting Thyroid Cytopathology has improved the quality of FNA reporting, promoting greater transparency and fewer unwarranted thyroidectomies (7).

However, Bethesda Category III ("atypia of unknown significance" [AUS], also termed "follicular lesion of unknown significance" [FLUS]) has generated controversy due to inconsistent usage across different clinicians and institutions. AUS/FLUS was defined for use as a category of last resort, with the expectation that $7 \%$ or fewer of FNAs would receive this diagnosis $(5,6)$. The Bethesda consensus publication estimates that such a nodule would be associated with a low risk of malignancy (5-15\%) and, in the absence of other suspicious features, could be managed with a repeat FNA. Conversely, Bethesda Category IV (follicular neoplasm or suspicious for follicular neoplasm) is thought to warrant surgery due to an estimated 15-30\% risk of malignancy. In contrast to the Bethesda recommendations, the guidelines released by the American Association of Clinical Endocrinologists/Associazione Medici Endocrinologi/European Thyroid Association (AACE/AME/ETA) Task Force combine Bethesda Categories III and IV (both defined as indeterminate) $(8,9)$. This group recommends surgery in most instances, with observation only in cases with favorable clinical, cytologic, and sonographic features.

The 5-15\% risk of malignancy from Bethesda Category III nodules remains an initial approximation, with the precise incidence variable among institutions $(5,6,10,11)$. The emergence and FDA approval of molecular testing also suggests the need to reexamine this percentage and its associated recommendation. The actual risk of malignancy is difficult to determine, since a pathologic diagnosis is only available in the subset of patients selected for surgery. Previous studies have not strictly followed Bethesda guidelines, have included incidental carcinomas in rates of malignancy, or have retrospectively classified specimens under the Bethesda System for Reporting Thyroid Cytopathology (12-16). Here, we describe the experience of a large cohort of patients with Bethesda Category III (AUS/FLUS) at a comprehensive cancer center, where thyroid nodules were classified at the time of care under the Bethesda System for Reporting Thyroid Cytopathology.

\section{Methods}

We reviewed the records of all patients with thyroid nodules classified by the Bethesda System between 2008 and 2011 at Memorial Sloan-Kettering Cancer Center (MSKCC). This includes FNAs performed at the MSKCC or outside FNAs interpreted by MSKCC cytopathologists. All patients had thyroid ultrasound records available. AUS/ FLUS patients with additional biopsy-proven thyroid malignancy, or previous FNAs demonstrating Bethesda Class IV-VI were excluded, as were those without documented follow-up. This study was deemed exempt by the MSKCC Institutional Review Board.

FNAs were performed by a dedicated radiologist using a 25- or 27-gauge needle under ultrasound guidance, typically with one to four passes. An air-dried slide was stained with a modified Giemsa stain for adequacy assessment. The remaining slides were alcohol-fixed and stained with hematoxylin and eosin. The needle was then rinsed in CytoLyt (Hologic, Marlborough, MA), and Papanicolaou-stained
ThinPrep slides were prepared using the ThinPrep 2000 (Hologic, Marlborough, MA). Cell block preparations were made when adequate material was available. On-site assessment for adequacy was routinely performed.

All reports followed the diagnostic scheme proposed by the 2007 National Cancer Institute Thyroid FNA State of the Science Conference Guidelines (10) and the subsequent Bethesda System for Reporting Thyroid Cytopathology (5). Additional cytologic substratification at our institution was also performed (17). Operative criteria included large multinodular goiter, concerning sonographic features (17), enlarging nodule size, clinical suspicion, or patient/physician preference. Patients with two consecutive AUS/FLUS diagnoses were recommended to undergo surgery for diagnostic and therapeutic purposes.

For patients undergoing surgery, AUS/FLUS nodules were identified by correlating cytology reports with ultrasound and pathology reports. For pathologic analysis, tumor size was considered the maximum diameter within the resected gland. Mitotic rate was ascertained by counting 10 high-power fields $(400 \times)$ with a U-DO microscope (Olympus, Center Valley, PA). Incidental carcinomas (including microcarcinomas) distinct from the target nodule were categorized and analyzed separately.

Estimates of the rate of malignancy in thyroid nodules are subject to several biases, as not all nodules undergo surgical resection and confirmatory pathologic analysis. Therefore, we determined the conceivable range of malignancy rates. The lower-bound estimate was calculated by dividing the number of confirmed malignancies by the total number of AUS/FLUS nodules, whether triaged to surgery, repeat FNA, or observation. The assumption that all observed (nonoperated) AUS/FLUS nodules were benign is subject to verification bias, and therefore underestimates the prevalence of malignancy. The upper-bound estimate was calculated by dividing the number of confirmed malignancies by the number of AUS/FLUS nodules selected to undergo surgery. As nodules selected for surgery may have other clinical or ultrasonographic features that increase suspicion, this number is subject to selection bias, overestimating the prevalence of malignancy. The true prevalence is likely to lie between the lower- and upper-bound approximations.

Clinical and ultrasonographic variables were recorded and evaluated for their association with surgery, including age, sex, size, irregular margins, hypoechogenicity, hypervascularity, and calcifications. Logistic regression analysis was performed using SPSS v21 (IBM Corp., Armonk, NY). Comparative analyses were performed with chi-square analysis with Yates correction. All $p$-values were predetermined to be two-sided, with the level of significance set at $p<0.05$. Confidence intervals (CI) were calculated using the Poisson distribution.

\section{Results}

Collectively, $8.0 \%$ (709/8862) of all thyroid FNAs performed during the study period were classified as AUS/FLUS, approximating Bethesda System for Reporting Thyroid Cytopathology guidelines (Table 1 and Fig. 1). Of the 709 patients with AUS/FLUS on initial FNA, 541 had documented follow-up, with a median period of 14 months. The median age of AUS/FLUS patients was 54.0 years, with $80.4 \%$ of patients 
TABle 1. COHORT FNA Classification by Bethesda Category

\begin{tabular}{lcr}
\hline Bethesda Category & $\begin{array}{c}\text { Bethesda } \\
\text { expected } \\
\text { incidence }\end{array}$ & $\begin{array}{c}\text { Cohort } \\
\text { incidence }\end{array}$ \\
\hline I (unsatisfactory) & $<10 \%$ & $3.2 \%$ \\
II (benign) & $60-70 \%$ & $72.9 \%$ \\
III (AUS/FLUS) & $<7 \%$ & $8.0 \%$ \\
IV (suspicious for & $\mathrm{N} / \mathrm{A}$ & $2.8 \%$ \\
$\quad$ follicular neoplasm) & $\mathrm{N} / \mathrm{A}$ & $4.6 \%$ \\
V (suspicious for malignancy) & $3-7 \%$ & $8.5 \%$ \\
VI (malignant) & & \\
\hline
\end{tabular}

AUS/FLUS, atypia of undetermined significance/follicular lesion of undetermined significance; FNA, fine-needle aspiration; N/A, not applicable.

being female. The median nodule size was $1.9 \mathrm{~cm}$. From this cohort, $64.7 \%$ (350/541) underwent immediate surgery, $17.7 \%$ (96/541) underwent repeat FNA, and $17.6 \%$ (95/541) were observed. The mean nodule sizes for these subgroups were $2.3 \mathrm{~cm}, 2.0 \mathrm{~cm}$, and $1.9 \mathrm{~cm}$ respectively.

On ultrasound, $11.5 \%$ of nodules were complex, $12.4 \%$ harbored microcalcifications, $19.4 \%$ had hypervascular features, and $21.4 \%$ were hypoechoic. In our cohort, $9.9 \%$ (51/ 541 ) of nodules were identified as positron emission tomography/computed tomography (PET/CT) scan incidentalomas, of which $13.7 \%$ (7/51) were found to be malignant. Additionally, $61.9 \%(335 / 541)$ were outside referrals $(29.6 \%$ were malignant), while $38.1 \%$ (206/541) were diagnosed internally at MSKCC (21.8\% were malignant). Substratification of AUS/FLUS nodules based on cytologic features is listed in Table 2.
Repeat FNA is recommended by the Bethesda System for Reporting Thyroid Cytopathology to help delineate clinical decision making in AUS/FLUS nodules (Table 3). Of the 96 nodules undergoing repeat FNA, the second FNA was unsatisfactory (Category I) in 5.2\% (5/96), benign (Category II) in $42.7 \%$ (41/96), AUS/FLUS (Category III) in $38.5 \%$ (37/ 96), suspicious for neoplasm (Category IV) in 5.2\% (5/96), suspicious for malignancy (Category V) in $4.2 \%$ (4/96), and malignant (Category VI) in 4.2\% (4/96; Fig. 2). Of those with a benign FNA who still underwent surgery (2/41), 0\% (0/2) were malignant. Of those nodules with two consecutive AUS/ FLUS diagnoses, $51.4 \%$ (19/37) went on to surgery and $26.3 \%(5 / 19)$ were found to be malignant.

On univariate logistic regression analysis, factors associated with triage to surgery were increasing nodule size $(p<0.0001)$, younger patient age $(p<0.0001)$, and presence of hypervascularity $(p=0.032)$. Sex, hypoechogenicity, infiltrative margins, and calcifications were not found to be predictive. On multivariable analysis, none of the above variables was found to be significant.

\section{Risk of malignancy among patients with AUS/FLUS nodules triaged to surgery (upper-bound estimate)}

Of the $350(64.7 \%)$ nodules with AUS/FLUS cytopathology that went on to immediate surgery, the rate of malignancy was $38.6 \%$ (135/350). Including the 31 nodules that were resected (after repeat FNA), the rate of malignancy for all patients triaged to surgery was 37.8\% (144/ 381) [CI 33.1-42.8]. These estimates represent the upper bound of the true incidence rate. The vast majority of malignant surgical specimens comprised papillary thyroid carcinoma, either classical or other subtypes $(86.8 \%)$. Follicular variant of papillary thyroid carcinoma comprised

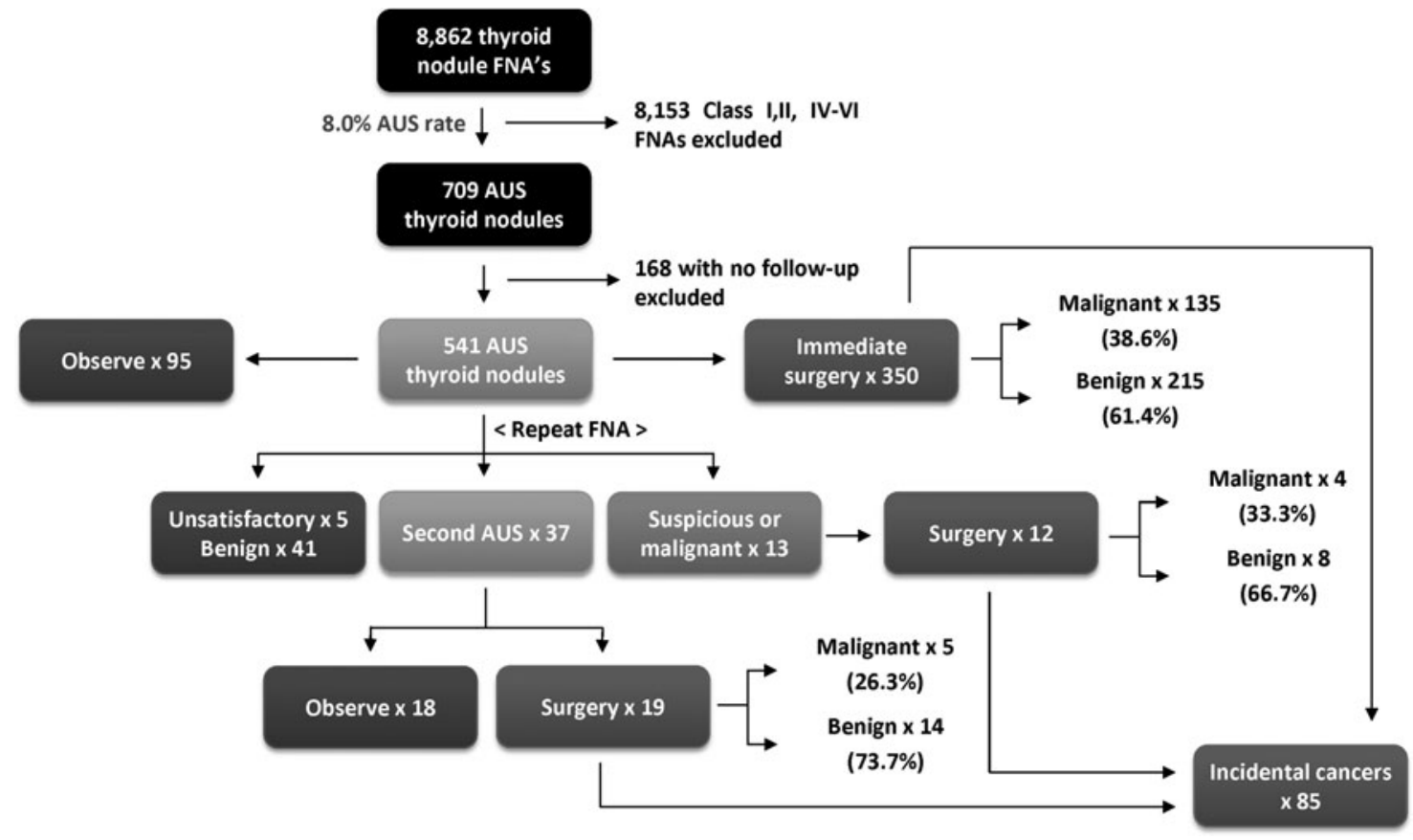

FIG. 1. Flow schematic of atypia of undetermined significance/follicular lesion of undetermined significance (AUS/ FLUS) thyroid nodule patients managed between 2008 and 2011. Malignancy rates shown are specific to the targeted AUS/ FLUS nodule, excluding incidental cancers or concurrent nodules. 
Table 2. Cytologic Substratification of Bethesda III Nodules

\begin{tabular}{lcr}
\hline AUS/FLUS cases with surgery & \multicolumn{1}{c}{ Benign $^{\text {a }}$} & \multicolumn{1}{c}{ Malignant $^{-}$} \\
\hline AUS/FLUS—NOS $(n=218)$ & $41.3 \%$ [CI 36.0-46.6] & $36.2 \%$ [CI 29.4-42.5] \\
AUS/FLUS—favor benign $(n=13)$ & $76.9 \%$ [CI 69.2-84.6] & $7.7 \%$ [CI 1.9-36.0] \\
AUS/FLUS—cannot exclude PTC $(n=28)$ & $28.6 \%$ [CI 9.4-47.8] & $53.6 \%$ [CI 33.9-72.1] \\
AUS/FLUS—cannot exclude Hürthle cell neoplasm $(n=44)$ & $34.1 \%$ [CI 20.5-47.7] & $29.5 \%$ [CI 16.8-45.2] \\
AUS/FLUS—cannot exclude follicular neoplasm $(n=78)$ & $24.4 \%$ [CI 11.9-36.9] & $56.4 \%$ [CI 44.7-67.6] \\
\hline
\end{tabular}

${ }^{a}$ Excludes nonmalignant neoplasms.

CI, confidence interval; PTC, papillary thyroid carcinoma; NOS, not otherwise specified.

$48.8 \%$ of the papillary thyroid cancers. The majority of benign surgical specimens consisted of nodular hyperplasia (51.9\%; Fig. 3).

There were an additional 85 patients with separate incidental thyroid cancers identified, 76 of which were papillary microcarcinomas $(89.4 \%)$. There were 11 cases with dual primary and incidental cancers. If incidental cancers are included, the cumulative rate of malignancy in resected glands (counting AUS/FLUS cancerous nodules and incidental carcinomas) was $57.2 \%(218 / 381)$.

\section{Risk of malignancy among all patients with AUS/FLUS nodules (lower-bound estimate)}

The incidence of malignancy among all patients with a nodule classified as AUS/FLUS, whether triaged to surgery or not, was $26.6 \%(144 / 541)$ [CI 22.4-31.3]. This estimate represents the lower bound of the true incidence rate, as it assumes that all observed nodules are benign.

\section{Risk of malignancy among patients with consecutive AUS/FLUS FNAS}

The upper-bound estimate of patients with two successive FNAs showing AUS/FLUS was 26.3\% (5/19). Assuming the observed patients had benign nodules, the lower-bound estimate was $13.5 \%(5 / 37)$.

\section{Discussion}

The Bethesda System for Reporting Thyroid Cytopathology has greatly facilitated the standardization of FNA reporting. By clarifying ambiguous language and riskstratifying patients, it has enabled more precise decision making when selecting patients for surgery. Its rationale is also based upon multidisciplinary consensus and a reasonable incidence of malignancy. For instance, the recommendation for clinical follow-up for Bethesda Category II (benign) is based on a $0-3 \%$ risk of malignancy, while the recommendation for surgery for Bethesda Category V (suspicious for malignancy) is based on a $60-75 \%$ risk $(5,6)$.

However, the appropriate level of risk to assign to Bethesda Category III (AUS/FLUS) nodules remains ambiguous, with some arguing against the need for equivocation in a system designed to minimize it $(4,14)$. Shi et al. found that eliminating the use of AUS/FLUS as a diagnosis considerably decreased FNA sensitivity, increasing false positive and false negative rates while escalating interand intra-observer variability (14). Nevertheless, the exact risk of malignancy has been unclear, as many AUS/FLUS nodules are observed with no pathologic confirmation available.

This study examined the histologic outcomes in a large number of AUS/FLUS nodule patients classified with Bethesda criteria and treated at a comprehensive cancer center. The heterogeneity of the AUS/FLUS diagnosis has led to this cytopathologic diagnosis being rendered at rates of up to $18 \%$ in some series $(12,16)$, suggesting high variability across institutions. Unlike previous reports, the incidence of AUS/FLUS diagnoses in this study was similar to the Bethesda System for Reporting Thyroid Cytopathology recommended rate of $7 \%$, indicating that the results are comparable with its intent. As a high percentage of patients underwent immediate surgery after FNA, this cohort distinctively provides pathological confirmation for nodules with an initial AUS/FLUS diagnosis.

Before proceeding, it is important to mention a number of caveats pertaining to our data. Any estimate of the risk of malignancy in indeterminate thyroid nodules is limited by the fact that not all nodules undergo surgery with resultant pathologic analysis. There are a number of factors, described in detail below, that may influence the decision to refer a patient with a Bethesda III thyroid nodule to a specific

Table 3. Results of Second Fine-Needle Aspiration After Initial AUS/Flus Diagnosis

\begin{tabular}{lccc}
\hline Bethesda Category & Second FNA (n) & Surgery (n) & Cancer in specimen $(\mathrm{n})$ \\
\hline I (unsatisfactory) & $5(5.2 \%)$ & $2(40.0 \%)$ & $0(0.0 \%)$ \\
II (benign) & $41(42.7 \%)$ & $2(4.9 \%)$ & $0(0.0 \%)$ \\
III (AUS/FLUS) & $37(38.5 \%)$ & $19(51.3 \%)$ & $5(26.3 \%)$ \\
IV (suspicious for follicular neoplasm) & $5(5.2 \%)$ & $4(80.0 \%)$ & $2(50.0 \%)$ \\
V (suspicious for malignancy) & $4(4.2 \%)$ & $2(50.0 \%)$ & $0(0.0 \%)$ \\
VI (malignant) & $4(4.2 \%)^{\mathrm{a}}$ & $2(50.0 \%)^{\mathrm{b}}$ & $2(100.0 \%)$ \\
Total & $\mathbf{9 6}$ & $\mathbf{3 1}$ & $\mathbf{9}$ \\
\hline
\end{tabular}

${ }^{\mathrm{a}}$ Papillary thyroid cancer $\times 3$, lymphoma $\times 1$.

$b_{2}$ patients (lymphoma, papillary thyroid cancer on FNA) did not undergo surgery. 


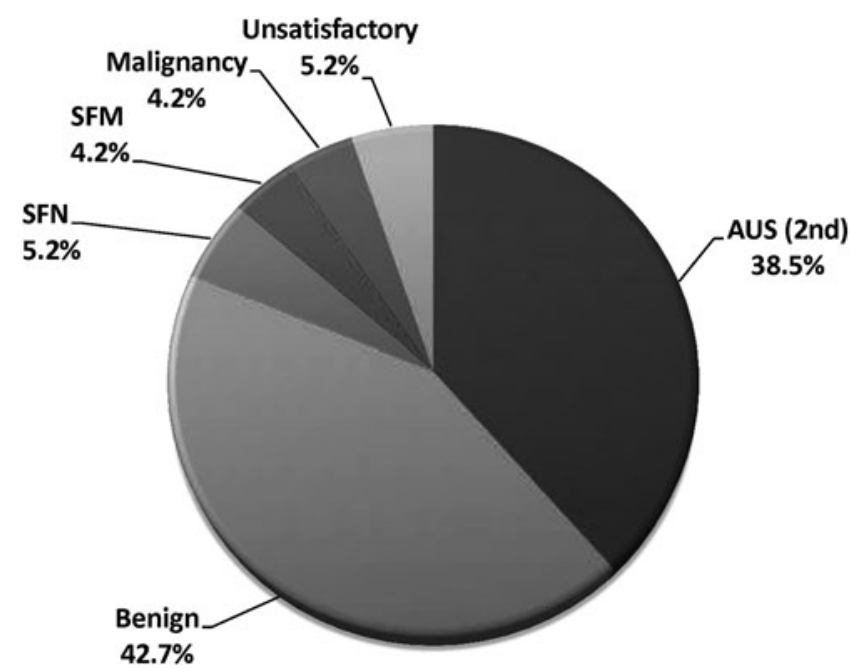

FIG. 2. Repeat fine-needle aspiration (FNA) results after initial AUS/FLUS diagnosis. A second (consecutive) AUS/ FLUS diagnosis was found in $38.5 \%$ of patients, while $42.7 \%$ of repeat FNAs were benign. AUS/FLUS, Atypia of Undetermined Significance/Follicular Lesion of Undetermined Significance (Bethesda Category III); SFN, suspicious for neoplasm (Bethesda Category IV); SFM, suspicious for malignancy (Bethesda Category V).

institution and, furthermore, that may influence the decision to triage a patient to surgical resection. These types of "referral bias" and "selection bias," if present, tend to enrich the prevalence of malignancy within Bethesda III nodules. For these reasons, rather than calculating one malignancy rate, we have instead provided both lower- and upper-bound estimates of the prevalence of malignancy in this cohort. Furthermore, we have carefully examined the characteristics of our cohort, and not found evidence of disproportionate bias toward the higher-risk end of the spectrum. Nevertheless, the rate of malignancy in Bethesda III nodules does vary among institutions, and is likely to be higher at a referral center such as ours.

Accordingly, we estimate that the risk of malignancy for an AUS/FLUS nodule is between $26.6 \%$ and $37.8 \%$, notably higher than the 5-15\% approximation. Such numbers may be elevated enough to justify surgery, not unlike the recommendation for lobectomy based upon Bethesda Category IV risk (15-30\%; Fig. 4). Collectively, our results suggest that
AUS/FLUS nodules, even at the low "last resort" incidence suggested by the Bethesda System for Reporting Thyroid Cytopathology, may warrant reconsideration of current recommendations. However, we caution that our results may not be generalizable to AUS/FLUS cohorts substantially different from that described here.

Other published cohorts of smaller size have suggested wide-ranging malignancy risk for AUS/FLUS nodules. Parengi et al. observed an incidence of 16\% [CI 10.2-23.6] (18), while VanderLaan et al. reported a prevalence of $46 \%$ [CI 36.8-56.1] (16). These and other outcomes have been confounded by study periods predating the Bethesda System for Reporting Thyroid Cytopathology, an incidence of AUS/ FLUS diagnoses that is higher than the $7 \%$ expected, and, in some cases, inflation of the malignancy rate with incidental cancers (12-16). Differences may also relate to random variation or institutional differences in pathologic interpretation. Nonetheless, the confidence intervals of these series largely overlap those of the current study, further supporting the higher than expected cancer incidence in AUS/FLUS.

The limitations of cytology underscore the importance of emerging molecular assays in determining the need for surgical intervention. Recent advancements in a gene expression classifier assay using FNA material have demonstrated high negative predictive value in cytologically indeterminate thyroid nodules (Bethesda Classes III-V) (19). As our study supports that the pretest probability of malignancy for AUS/ FLUS nodules is $>5-15 \%$, such molecular testing may become instrumental to rule out cancer judiciously and reduce unnecessary thyroidectomies. However, a higher pretest probability of malignancy, such as was observed in this cohort, may limit the utility of certain molecular classifiers to reliably determine benignancy (20). It is therefore critical to estimate the rates of malignancy at each insititution.

Although the Bethesda System for Reporting Thyroid Cytopathology recommends repeat FNA for AUS/FLUS, the American Thyroid Association (ATA) is less clear (17), while the AACE/AME/ETA guidelines recommend against repeat FNA due to the possibility of confounding results $(8,9)$. In this study, the incidence of malignancy after one AUS/FLUS diagnosis was notably no different from the incidence of cancer after two $(p=0.98)$, corresponding to findings by VanderLaan et al. (16). However, the repeat FNA was not indeterminate in $56.3 \%$ (54/96) of our patients, raising the question of whether the Bethesda recommendation for repeat FNA may be beneficial in some cases. The

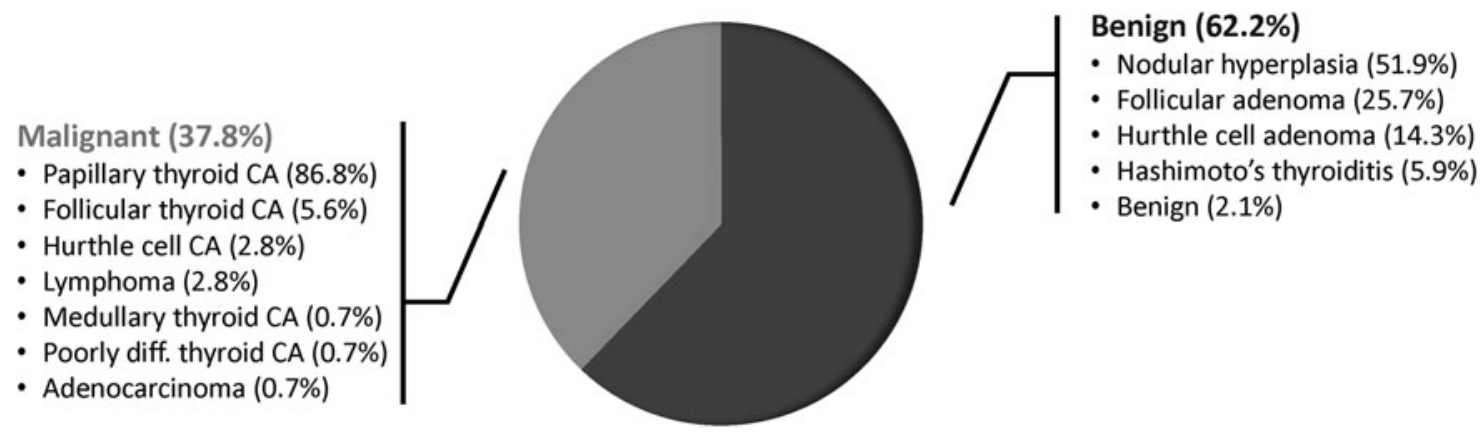

FIG. 3. Histologic outcomes for AUS/FLUS patients triaged to surgery. AUS/FLUS nodules with a malignant diagnosis most commonly harbored papillary thyroid carcinoma. CA, cancer; diff., differentiated. 


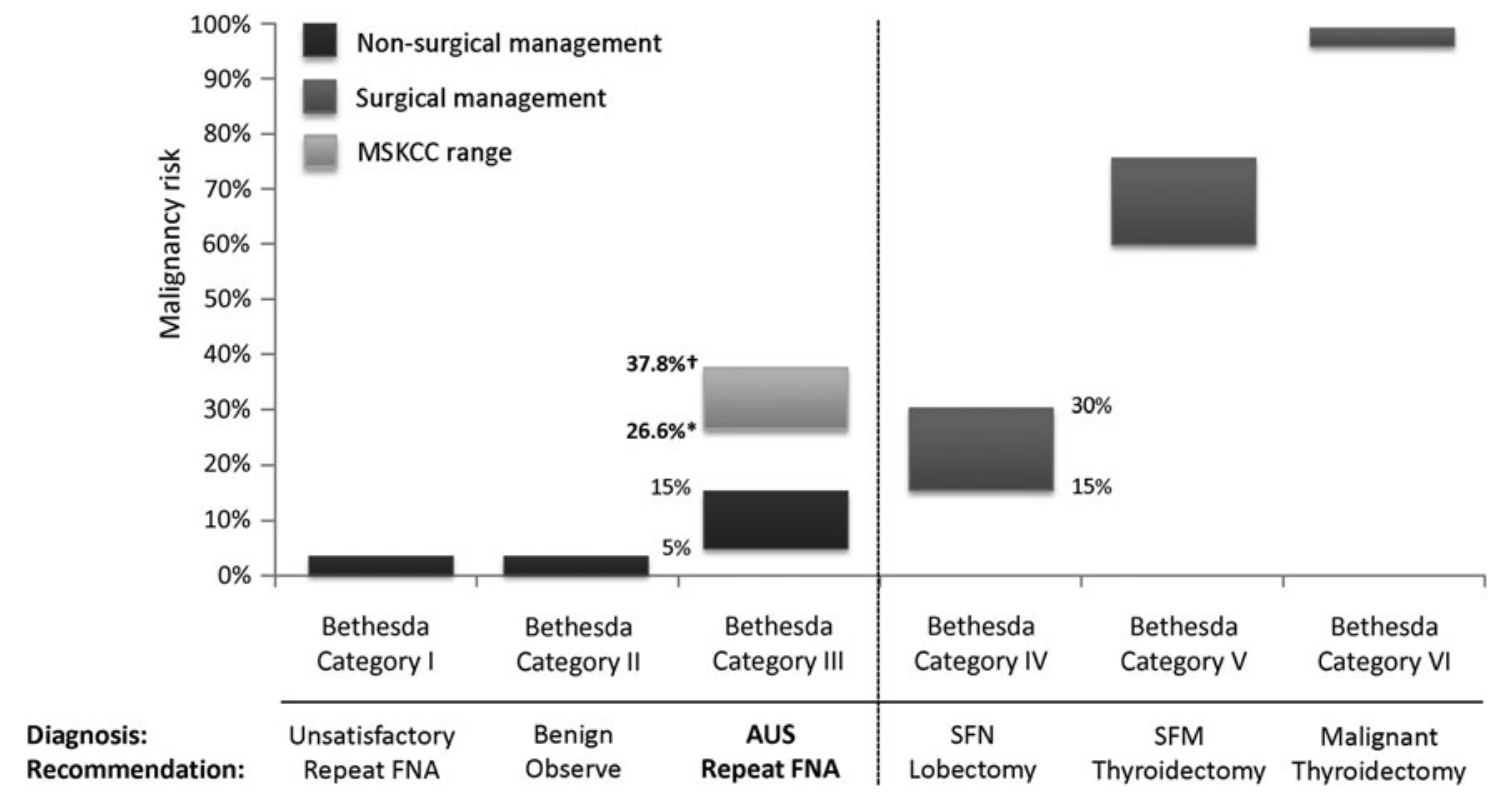

FIG. 4. Adjusted malignancy risk of thyroid nodules by Bethesda classification. The incidence of malignancy for AUS/ FLUS observed is higher than that estimated by the Bethesda System for Reporting Thyroid Cytopathology, and falls within the range where surgical intervention is recommended (Bethesda Category IV). *Conservative estimate assuming all observed/nonsurgical patients are benign. ${ }^{\dagger}$ Aggressive estimate considering only nodules triaged to surgery. MSKCC, Memorial Sloan-Kettering Cancer Center; SFN, suspicious for neoplasm; SFM, suspicious for malignancy.

accuracy of the diagnosis rendered on repeat FNA may not be clear. In the two series by Vanderlaan et al. and Renshaw et al., patients with a Bethesda Category III FNA followed by a benign FNA (and who went on to surgery) had malignant disease in $9.4 \%(3 / 32)$ and $28.5 \%(2 / 7)$ of cases respectively $(16,21)$. In our series, $0 \%(0 / 2)$ harbored malignancy, but in actuality most of these patients (39/41) never went on to surgery. Given the small numbers in these subsets, the issue of repeat FNA remains of uncertain value: it is not clear whether a benign diagnosis on repeat FNA should be weighted more or less heavily than the index AUS/FLUS diagnosis. The risk of malignancy in a nodule with separate needle biopsies interpreted as AUS/FLUS and then benign may lie somewhere in between the risk of malignancy for the respective individual categories.

Substratification of thyroid nodules has generated growing interest (21-25), with potential implications for cytologybased risk stratification. Horne et al. subclassified 106 nodules into $\mathrm{IN}_{\mathrm{a}}$ (predominant microfollicular architecture; $7 \%$ malignancy rate) and $\mathrm{IN}_{\mathrm{b}}$ (nuclear atypia; $56 \%$ malignancy rate) categories (23). These results suggest that indeterminate nodules with nuclear atypia could be at substantially higher risk for malignancy. In our cohort, similar substratification was not informative: the risk of malignancy in "AUS/

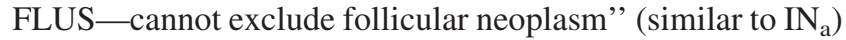
was 56.4\%, while "AUS/FLUS — cannot exclude PTC" ( similar to $\mathrm{IN}_{\mathrm{b}}$ ) risk was $53.6 \%$ (Table 2). Only $7.3 \%$ of nodules in our cohort had features corresponding to the higher-risk " $\mathrm{IN}_{\mathrm{b}}$ " category, suggesting this subgroup may be less common. The predominant subcategory identified in our study was "AUS/FLUS-not otherwise specified" (57.2\% of patients), which had a $36.2 \%$ malignancy rate.

Limitations of the current study include lack of extended follow-up, resulting in possible verification bias. Given the nature of AUS/FLUS management, some observed nodules may never show clinical signs of malignancy and yet may harbor cancer. This would lead our lower-bound estimate to be a significant underestimate of the prevalence of malignancy. The indolent nature of many thyroid cancers makes this scenario very possible. Conversely, the nodules with pathologic specimens for analysis are skewed due to the selection of those patients for surgery: resection could have been chosen due to unfavorable clinical or radiographic features that elevate the likelihood of malignancy. Although various sonographic factors are known predictors of malignancy (17), only hypervascularity, younger age, and increasing size were significant predictors of surgery on univariate analysis. Size in particular may be a major factor, as a $4 \mathrm{~cm}$ AUS/FLUS nodule will likely be managed differently from a $1 \mathrm{~cm}$ AUS/FLUS nodule. However, none of these was significant on multivariable analysis. Our institutional philosophy of triaging most AUS/FLUS patients to surgery may have superseded these factors. Nonetheless, Nagarkatti et al. similarly found that ultrasonographic features such as microcalcifications, irregular margins, and hypoechogenicity were not associated with a decision to perform surgery (18). Such findings suggest that imaging features predictive for malignancy in all thyroid nodules do not extend to those predictive for surgery, especially in an AUS/FLUS nodule subset.

Importantly, certain higher-risk AUS/FLUS subgroups may be enriched at a cancer center, with such a referral bias potentially inflating the malignancy rate. The elevated cancer incidence could also be ascribed to a lower diagnostic threshold whereby higher-risk Bethesda Categories (IV-VI) are called AUS/FLUS at our institution. The median age, sex breakdown, and median nodule size of patients in this MSKCC cohort are comparable to other reports $(19,23)$. For example, in the molecular classifier study by Alexander et al. (19) that studied 577 patients with indeterminate nodules 
(predominantly from community physicians), median age was 52.8 years (54.0 years in this cohort), $78.2 \%$ were female ( $80.4 \%$ in this cohort), and median nodule size was $2.2 \mathrm{~cm}$ $(1.9 \mathrm{~cm}$ in this cohort). Analysis of all FNAs performed at our institution during the study period found that the overall breakdown of Bethesda Categories was not skewed toward higher-risk categories (Table 1). Moreover, within the AUS/ FLUS cohort itself, the higher-risk subgroups with a higher probability of cancer ("AUS/FLUS - cannot rule out PTC," corresponding to the $\mathrm{IN}_{\mathrm{b}}$ category of Horne et al. (23)) comprised only $7.3 \%$ of our cohort (Table 2 ). The incidence of PET/CT incidentalomas, which may reflect a higher rate of malignancy, was also not inordinately high (9.9\%), and the incidence of cancer within this PET/CT subgroup was modest $(13.7 \%)$. Nevertheless, we cannot definitely rule out the potential for other, unmeasured sources of bias, and therefore have deliberately described lower- and upperbound estimates to address such uncertainty. As the prevalence of malignancy may vary between institutions, we believe there is value to presenting our analysis with such a range.

\section{Conclusion}

The risk of malignancy in AUS/FLUS thyroid nodules as defined by the Bethesda System for Reporting Thyroid Cytopathology is higher than estimated, with an estimated prevalence of 26.6-37.8\%. The incidence of malignancy after an index AUS/FLUS diagnosis is also similar to the rate after consecutive AUS/FLUS diagnoses, suggesting that repeat FNA may not have clear utility in clinical decision making. The limitations of FNA cytology imply that other diagnostic options, including molecular assays, may be valuable to delineate risk further and assist clinicians in identifying low-risk patients who may not require surgery.

\section{Author Disclosure Statement}

The authors declare no competing commercial or financial conflicts of interest.

\section{References}

1. Yassa L, Cibas ES, Benson CB, Frates MC, Doubilet PM, Gawande AA, Moore FD Jr, Kim BW, Nose V, Marqusee E, Larsen PR, Alexander EK 2007 Long-term assessment of a multidisciplinary approach to thyroid nodule diagnostic evaluation. Cancer 111:508-516.

2. Powsner SM, Costa J, Homer RJ 2000 Clinicians are from Mars and pathologists are from Venus. Arch Pathol Lab Med 124:1040-1046.

3. Redman R, Yoder BJ, Massoll NA 2006 Perceptions of diagnostic terminology and cytopathologic reporting of fine-needle aspiration biopsies of thyroid nodules: a survey of clinicians and pathologists. Thyroid 16:1003-1008.

4. Somma J, Schlecht NF, Fink D, Khader SN, Smith RV, Cajigas A 2010 Thyroid fine needle aspiration cytology: follicular lesions and the gray zone. Acta Cytol 54:123-131.

5. Cibas ES, Ali SZ 2009 The Bethesda System for Reporting Thyroid Cytopathology. Thyroid 19:1159-1165.

6. Cibas ES, Ali SZ, Conference NCITFSotS 2009 The Bethesda System for Reporting Thyroid Cytopathology. Am J Clin Pathol 132:658-665.
7. Crowe A, Linder A, Hameed O, Salih C, Roberson J, Gidley J, Eltoum IA 2011 The impact of implementation of the Bethesda System for Reporting Thyroid Cytopathology on the quality of reporting, "risk" of malignancy, surgical rate, and rate of frozen sections requested for thyroid lesions. Cancer Cytopathol 119:315-321.

8. Gharib H, Papini E, Paschke R, Duick DS, Valcavi R, Hegedus L, Vitti P, Nodules AAETFoT 2010 American Association of Clinical Endocrinologists, Associazione Medici Endocrinologi, and European Thyroid Association medical guidelines for clinical practice for the diagnosis and management of thyroid nodules. J Endocrinol Invest 33:1-50.

9. Gharib H, Papini E, Paschke R, Duick DS, Valcavi R, Hegedus L, Vitti P, Nodules AAETFoT 2010 American Association of Clinical Endocrinologists, Associazione Medici Endocrinologi, and European Thyroid Association Medical guidelines for clinical practice for the diagnosis and management of thyroid nodules: executive summary of recommendations. Endocr Pract 16:468-475.

10. Baloch ZW, Cibas ES, Clark DP, Layfield LJ, Ljung BM, Pitman MB, Abati A 2008 The National Cancer Institute Thyroid fine needle aspiration state of the science conference: a summation. Cytojournal 5:6.

11. Ali SZ CE 2010 The Bethesda System for Reporting Thyroid Cytopathology. Springer, New York.

12. Nayar R, Ivanovic M 2009 The indeterminate thyroid fineneedle aspiration: experience from an academic center using terminology similar to that proposed in the 2007 National Cancer Institute Thyroid Fine Needle Aspiration State of the Science Conference. Cancer 117:195-202.

13. Layfield LJ, Morton MJ, Cramer HM, Hirschowitz S 2009 Implications of the proposed thyroid fine-needle aspiration category of "follicular lesion of undetermined significance"': a five-year multi-institutional analysis. Diagn Cytopathol 37:710-714.

14. Shi Y, Ding X, Klein M, Sugrue C, Matano S, Edelman M, Wasserman P 2009 Thyroid fine-needle aspiration with atypia of undetermined significance: a necessary or optional category? Cancer 117:298-304.

15. Jo VY, Stelow EB, Dustin SM, Hanley KZ 2010 Malignancy risk for fine-needle aspiration of thyroid lesions according to the Bethesda System for Reporting Thyroid Cytopathology. Am J Clin Pathol 134:450-456.

16. VanderLaan PA, Marqusee E, Krane JF 2011 Clinical outcome for atypia of undetermined significance in thyroid fine-needle aspirations: should repeated FNA be the preferred initial approach? Am J Clin Pathol 135:770775.

17. Cooper DS, Doherty GM, Haugen BR, Kloos RT, Lee SL, Mandel SJ, Mazzaferri EL, McIver B, Pacini F, Schlumberger M, Sherman SI, Steward DL, Tuttle RM; American Thyroid Association Guidelines Taskforce on Thyroid Nodules and Differentiated Thyroid Cancer 2009 Revised American Thyroid Association management guidelines for patients with thyroid nodules and differentiated thyroid cancer. Thyroid 19:1167-1214.

18. Nagarkatti SS, Faquin WC, Lubitz CC, Garcia DM, Barbesino G, Ross DS, Hodin RA, Daniels GH, Parangi S 2013 Management of thyroid nodules with atypical cytology on fine-needle aspiration biopsy. Ann Surg Oncol 20:60-65.

19. Alexander EK, Kennedy GC, Baloch ZW, Cibas ES, Chudova D, Diggans J, Friedman L, Kloos RT, LiVolsi 
VA, Mandel SJ, Raab SS, Rosai J, Steward DL, Walsh PS, Wilde JI, Zeiger MA, Lanman RB, Haugen BR 2012 Preoperative diagnosis of benign thyroid nodules with indeterminate cytology. New Engl J Med 367:705715 .

20. McIver B 2013 Evaluation of the thyroid nodule. Oral Oncol 49:645-653.

21. Renshaw AA 2010 Should "atypical follicular cells" in thyroid fine-needle aspirates be subclassified? Cancer Cytopathol 118:186-189.

22. VanderLaan PA, Marqusee E, Krane JF 2011 Usefulness of diagnostic qualifiers for thyroid fine-needle aspirations with atypia of undetermined significance. Am J Clin Pathol 136:572-577.

23. Horne MJ, Chhieng DC, Theoharis C, Schofield K, Kowalski D, Prasad ML, Hammers L, Udelsman R, Adeniran AJ 2012 Thyroid follicular lesion of undetermined significance: evaluation of the risk of malignancy using the two-tier sub-classification. Diagnostic Cytopathol 40: 410-415.

24. Walts AE, Mirocha J, Bose S 2014 Follicular lesion of undetermined significance in thyroid FNA revisited. Diagnostic Cytopathol 42:18-22.

25. British Thyroid Association 2007 Guidelines for the Management of Thyroid Cancer. Report of the Thyroid Cancer Guidelines Update Group. Second edition. Royal College of Physicians, London.

Address correspondence to: Luc G.T. Morris, MD, MSc

Head and Neck Service, Department of Surgery Memorial Sloan-Kettering Cancer Center 1275 York Avenue, Box 240 New York, NY 10065

E-mail: morrisl@mskcc.org 